\title{
Effect of surface tension for nanomaterial under normal triangle distribution force
}

\author{
Liyuan Wang ${ }^{1,2}$ \\ ${ }^{1}$ Regional Circular Economy key Laboratory of Gansu Higher Institutions; \\ ${ }^{2}$ Department of Mathematics, Longqiao College of Lanzhou University of Finance and Economics, \\ Lanzhou 730101, China. \\ wly811220@163.com
}

Keywords: Contact, surface tension, nanomaterial, normal triangle distribution force.

\begin{abstract}
Consideration the effects of surface tension on the elastic field of solid material has applications in several modern problems in solid mechanics. This paper proposes an application of surface elasticity theory in the analysis of contact problem at nanoscale. The Fourier integral transform method is adopted to derive the fundamental solutions for contact problem with surface tension effects. As a special case, the deformation induced by normal triangle distribution force is discussed in detail. The results indicate some interesting characteristics in nano-mechanics, which are distinctly different from those in classical contact problem.
\end{abstract}

\section{Introduction}

In the recent years, many new nano-materials have been developed by utilizing the fact that, at a nano-scale, materials begin to exhibit unique mechanical properties, which significantly differ from those at a larger scale.

The surface of solids is a special region with very small thickness (a few times of atom-spacing). Since the equilibrium lattice spacing in the surface is different from that in the bulk, surface effect appears. For solids with large characteristic dimensions, the volume ratios of surface region to the bulk material is small, the effect of surface then can be neglected because of its relatively tiny contribution. However, for micro-nano solids with large surface-to-bulk ratio the significance of surfaces is likely to be important. Form the viewpoint of continuum mechanics, this difference can be described by such concepts as surface tension, surface energy, and surface constitutive relations [1]. This is extremely true for nano-scale materials or structures. In such cases, the surface residual tension plays a critical role and thus has been adding its appeal to many researchers. For example, Miller and Shenoy [2] first probed the size-dependent elastic properties of nanoplates and beams. Sharma et al. [3] and Sharma and Ganti [4] studied the effect of surface/interfacial energy on the Eshelby's inclusion and inhomogeneity problems. For more recent developments in this field, the readers can refer to a review article by Wang et al. [5].

\section{Nanocontact model with surface tension}

In the absence of body force, the equilibrium equations, constitutive law, and geometry relations in the bulk are as follows

$$
\begin{aligned}
& \sigma_{i j, j}=0 \\
& \sigma_{i j}=2 G\left(\varepsilon_{i j}+\frac{\mu}{1-2 \mu} \varepsilon_{k k} \delta_{i j}\right)
\end{aligned}
$$

where $\mathrm{G}$ and $\mu$ are the shear modulus and Poisson's ratio of the bulk material, $\sigma_{i j}$ and $\varepsilon_{i j}$ are the stress tensor and strain tensor in the bulk material, respectively. Throughout the paper, Einstein's summation convention is adopted for all repeated Latin indices $(1,2,3)$ and Greek indices $(1,2)$. 
The strain tensor is related to the displacement vector $u_{i}$ by

$\varepsilon_{i j}=\frac{1}{2}\left(u_{i, j}+u_{j, i}\right)$

On the surface, the generalized Young-Laplace equation, surface constitutive relation and strain-displacement relationship can be expressed as

$$
\begin{aligned}
& \sigma_{\beta \alpha} n_{\beta}+\sigma_{\beta \alpha, \beta}^{s}=0 \\
& \sigma_{i j} n_{i} n_{j}=\sigma_{\beta \alpha}^{s} \kappa_{\beta \alpha} \\
& \sigma_{\beta \alpha}^{s}=\tau^{s} \delta_{\beta \alpha}+2\left(\mu^{s}-\tau^{s}\right) \varepsilon_{\beta \alpha}+\left(\lambda^{s}+\tau^{s}\right) \varepsilon_{\gamma \gamma} \delta_{\beta \alpha}+\tau^{s} u_{\beta, \alpha}^{s} \\
& \varepsilon_{\alpha \beta}^{s}=\frac{1}{2}\left(u_{\alpha, \beta}^{s}+u_{\beta, \alpha}^{s}\right)
\end{aligned}
$$

where $n_{i}$ denotes the normal to the surface, $\kappa_{\beta \alpha}$ the curvature tensor of the surface, $\sigma_{\alpha \beta}^{s}$ and $\varepsilon_{\alpha \beta}^{s}$ the surface stress and surface strain tensor, $\tau^{s}$ is the residual surface tension under unstrained conditions, $\mu^{s}$ and $\lambda^{s}$ are surface Lamé constants which can be determined by atom simulations or experiments [2].

As in classical theory of elasticity, the Airy stress function $\chi(x, z)$ is defined by

$$
\sigma_{x x}=\frac{\partial^{2} \chi}{\partial z^{2}}, \sigma_{z z}=\frac{\partial^{2} \chi}{\partial x^{2}}, \sigma_{x z}=-\frac{\partial^{2} \chi}{\partial x \partial z}
$$

Then the equilibrium equations in Eq. (6) are satisfied automatically, and the compatibility equation in Eq. (12) becomes

$$
\left(\frac{\partial^{2}}{\partial x^{2}}+\frac{\partial^{2}}{\partial z^{2}}\right)\left(\frac{\partial^{2} \chi}{\partial x^{2}}+\frac{\partial^{2} \chi}{\partial z^{2}}\right)=0
$$

To solve the boundary value problem, the Fourier integral transformation method is adopted to the coordinate $x$. Then, the Airy stress function $\chi(x, z)$ and its Fourier transformation $\tilde{\chi}(\xi, z)$ can be expressed as

$$
\begin{aligned}
& \tilde{\chi}(\xi, z)=\frac{1}{\sqrt{2 \pi}} \int_{-\infty}^{\infty} \chi(x, z) e^{i x \xi} d \xi \\
& \chi(\xi, z)=\frac{1}{\sqrt{2 \pi}} \int_{-\infty}^{\infty} \tilde{\chi}(x, z) e^{-i x \xi} d \xi
\end{aligned}
$$

Substituting Eqs. (8) into Eq. (7) and considering the condition that the stresses vanish at infinity, one obtains

$$
\tilde{\chi}(\xi, z)=(A+B z) e^{-z|\xi|} \text {. }
$$

where $A$ and $B$ are generally functions of $\xi$ as yet to be determined by boundary conditions.

Substituting Eq. (9) and Eqs. (8) into Eq. (7), the stresses can be written as

$$
\begin{aligned}
& \sigma_{z z}=-\frac{1}{\sqrt{2 \pi}} \int_{-\infty}^{\infty}[A(\xi)+z B(\xi)] \xi^{2} e^{-i x \xi-z|\xi|} d \xi \\
& \sigma_{x z}=\frac{i}{\sqrt{2 \pi}} \int_{-\infty}^{\infty} \xi[(1-|\xi| z) B(\xi)-|\xi| A(\xi)] e^{-i x \xi-z|\xi|} d \xi
\end{aligned}
$$

By substituting the stresses into the Eq. (7) and using Eqs. (8), the displacements are derived as

$$
w(x, z)=\frac{1}{2 G \sqrt{2 \pi}} \int_{-\infty}^{\infty}[|\xi| A(\xi)+(1-2 v+z|\xi|) B(\xi)] e^{-i x \xi-z|\xi|} d \xi+C_{2}
$$




\section{Elastic solution under normal triangle distribution force.}

As a particular example, let us consider the effect of a normal triangle distribution force $p(x)$ over the region $|x| \leq a$, while the shears force form zero $\left(O_{1}\right.$ and $\left.O_{2}\right)$ uniformly increased to maximum $p_{0}$ (O), while remainder of the boundary $y=0$ being unstressed.

$$
p(x)=\frac{p_{0}}{a}(a-|x|), \quad|x| \leq a
$$

If the change of the atomic spacing in deformation is infinitesimal, the contribution from the second term to the surface stresses is negligibly small compared to the residual surface tension [5]. In what follows, we keep only the first term in Eq. (4). Then, the surface stresses are given by

$$
\sigma_{\beta \alpha}^{s}=\tau^{s} \delta_{\beta \alpha}
$$

In this case, the boundary conditions (3) on the contact surface ( $z=0)$ are simplified to

$$
\begin{aligned}
& \sigma_{x z}(x)=0 \\
& p(x)+\sigma_{z z}(x)=-\frac{\tau^{s}}{R(x)}
\end{aligned}
$$

Substituting Eqs. (14) into Eqs. (9), one obtains

$$
B=A|\xi|
$$

On the surface, the radius of curvature due to deformation is given by

$$
\frac{1}{R(x)}=\frac{\partial^{2} w(x, 0)}{\partial x^{2}}
$$

By substituting Eqs. (15) and (16) into the surface condition Eqs. (14), $A(\xi)$ is determined by

$$
A(\xi)=\frac{\tilde{p}(\xi)}{(1+s|\xi|) \xi^{2}}
$$

where

$$
\begin{aligned}
& s=\frac{\tau^{s}(1-v)}{G} \\
& \tilde{p}(\xi)=\frac{p_{0}}{a} \sqrt{\frac{2}{\pi}} \frac{1-\cos (a \xi)}{\xi^{2}}
\end{aligned}
$$

Therefore $A(\xi)$ is given by

$$
A(\xi)=\frac{p_{0}}{a} \sqrt{\frac{2}{\pi}} \frac{1-\cos (a \xi)}{(1+s|\xi|) \xi^{4}}
$$

Substituting Eq. (20) into Eqs. (10) and (11), the stresses component and displaces component are obtained as

$$
\begin{aligned}
& \sigma_{z z}=-\frac{2 q_{0}}{\pi a} \int_{0}^{\infty}\left(\frac{z \xi+1}{1+b \xi}\right) \frac{\cos (x \xi)}{\xi^{2}}[1-\cos (a \xi)] e^{-z \xi} d \xi \\
& w(x, z)=\frac{p_{0}}{\pi G a} \int_{0}^{\infty} \frac{z \xi+2(1-v)}{\xi^{3}(1+b \xi)} \cos (x \xi)[1-\cos (a \xi)] e^{-z \xi} d \xi+C_{2}
\end{aligned}
$$

It is seen, when $s=0$, that is, the surface influence is ignored in Eqs. (21), the stresses of the half-plane are consistent with those in the classical elastic results [6].

On the contact surface $z=0$, the normal stress is given by

$$
\sigma_{z z}(x, 0)=-\frac{2 p_{0}}{\pi} \int_{0}^{\infty}\left(\frac{1-\cos t}{t^{2}}\right)\left(1+\frac{s}{a} t\right)^{-1} \cos \left(\frac{x}{a} t\right) d t
$$


If the normal displacement is $w$ specified to be zero at a distance $r_{0}$ on the contact surface, that is, $w\left(r_{0}, 0\right)=0$, the displacement on the surface is derived as

$$
w(x, 0)=\frac{2 p_{0}(1-v) a}{\pi G} \int_{0}^{\infty}\left(\frac{1-\cos (t)}{t^{3}}\right)\left(\frac{s}{a}+1\right)^{-1}\left[\cos \left(\frac{x}{a} t\right)-\cos \left(\frac{r_{0}}{a} t\right)\right] d t
$$

It is instructive to examine the influence of the surface tension on the stresses and displacements of the contact surface and compare them with those in classical contact problem.

As show in Fig.1, the distribution of the normal stress $\sigma_{z z}$ that is predicted by the classical theory experiences a singularity at the loading boundary and does not change smoothly; thus, this result appears unreasonable. If the surface tension is ignored $(s / a=0)$, the values of $\sigma_{z z}$ reduce to the classical values.

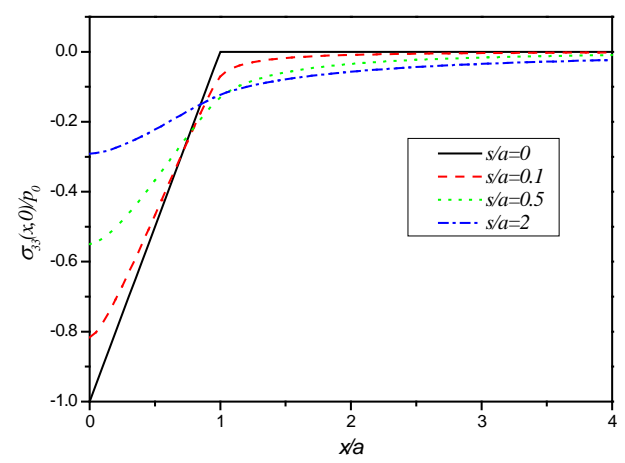

Fig.1 The distribution of contact normal stress $\sigma_{\mathrm{zz}}$ under normal triangle distribution load.

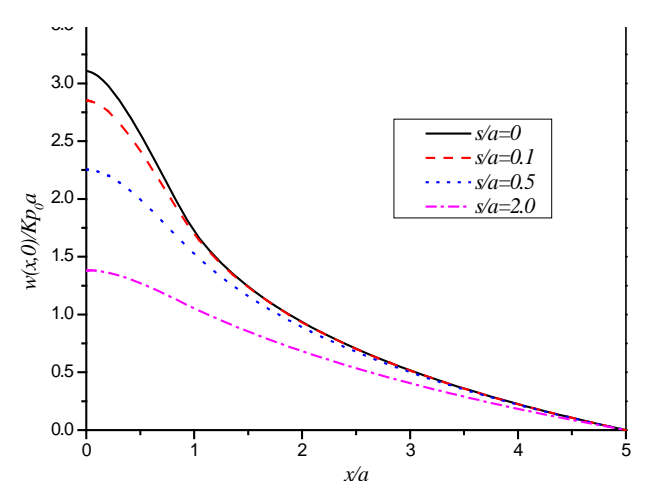

Fig.2 The distribution of surface indentation $w$ under normal triangle distribution load.

Due to the different residual surface stress value, the indent depth is plotted in Fig.2 with $K=2(1-v) / \pi G$, which also shows that the slope of the deformed surface for $a>0$ is continuous everywhere. It is also found the indent depth decreases with the increase of residual surface stress.

\section{Summary}

In this paper, we consider the two-dimensional contact problem in the light of surface elasticity theory. The general analytical solution is derived by using the Fourier integral transform method. For a particular loading case of normal triangle distribution force, the results are analyzed in detail and compared with the classical linear elastic solutions. A series of theoretical and numerical results show that the surface elasticity theory illuminates some interesting characteristics of contact problems at nano-scale, which are distinctly different from the classical solutions of elasticity without surface tension effects.

\section{References}

[1] R. C. Cammarata, "surface and interface stress effects in thin films” , Prog. Surface Sci, vol. 46, pp. 1-38, 1994.

[2] R. E. Miller and V. B. Shenoy, "Size-dependent elastic properties of nanosized structural elements”, Nanotechnology, vol. 11, no. 3, pp. 139-147, 2000.

[3] P. Sharma, S. Ganti and N. Bhate, "Effect of Surfaces on the Size-Dependent Elastic State of Nano-Inhomogeneities”, Appl. Phys. Lett., vol. 82,no. 4, pp. 535-537, 2003.

[4] P. Sharma and S. Ganti, “Size-Dependent Eshelby’s Tensor for Embedded Nano-Inclusions Incorporating Surface/Interface Energies” , ASME J. Appl. Mech., vol. 71,no. 5, pp. 663-671, 2004. 
[5] J. X. Wang, Z. P. Huang, H. L. Duan, S. W. Yu, X. Q. Feng, G. F. Wang, W. X. Zhang and T. J. Wang, "Surface Stress Effect in Mechanics of Nanostructured Materials", Acta Mech. Solida Sin., vol. 24, no.1, pp. 52-82. 2011.

[6] K. L. Johnson, ContactMechanics, CambridgeUniversity Press, Cambridge, Mass, USA, 1985. 